Original Article

\title{
The effects of Nordic and general walking on depression disorder patients' depression, sleep, and body composition
}

\author{
Seong Doo Park, PhD, $\mathrm{PT}^{1)}$, SeOng Hun Yu, PhD, $\mathrm{PT}^{2)^{*}}$ \\ 1) Department of Physical Therapy, Woosuk University Oriental Hospital, Republic of Korea \\ 2) Department of Physical Therapy, Gwang ju Trauma Center: 26 Sicheong-ro, Seo-gu, Gwang-ju \\ 500-827, Republic of Korea
}

\begin{abstract}
Purpose] This study examined Nordic walking as an exercise intervention for the elderly with depression. [Subjects] Twenty-four patients who were diagnosed with depression were randomly selected and divided into two groups, an experimental group which performed Nordic walking, and a control group, which performed normal walking. [Methods] Both groups practiced their respective walking exercise for 50 minutes per day, three times a week for eight weeks. To compare the effects of the intervention, psychological factors using the Beck depression inventory and sleep quality was assessed using the Korean version Pittsburgh sleep quality index. Skeletal muscle mass, fat free mass, body mass index, body fat percentage, and basal metabolism were estimated three times by a body composition analyzer, before the intervention, four weeks after the intervention, and eight weeks after the intervention. [Results] There was a significant difference in depression with a main effect of time in both groups. There was also a significant difference in sleep in over time and interaction. The differences over time between the two groups were significant for depression, sleep, and skeletal muscle mass. [Conclusion] The results suggests that Nordic walking has a positive effect on depression and sleeping disorders of the elderly, suggesting that Nordic walking based exercise programs should be developed for the elderly who suffer from depression or a sleeping disorder.

Key words: Depression, Nordic walking, Sleep
\end{abstract}

(This article was submitted Apr. 2, 2015, and was accepted Apr. 24, 2015)

\section{INTRODUCTION}

Elderly people experience rapid physical aging and deterioration of physical function as they get older ${ }^{1)}$. Depression in senescence, in particular, is known to not only decrease life satisfaction, but to also increase the potential mortality rate due to increased occurrence rate of physical disease and greater risk of suicide, if not properly treated ${ }^{2,3)}$.

In looking at the literature on elderly people's depression, depression shown throughout the senescence is closely related to the stress. Here, the stress due to old age is the direct cause of depression ${ }^{4}$. That is, stress coming from physical disease, death of a spouse, worsening financial situation, isolation from family and society, emigration, etc. can cause specific symptoms of depression ${ }^{5)}$.

Moreover, aged people frequently complain about health problems due to diminished sleep quality, despite unchanged length of sleeping time ${ }^{6}$. Worsening sleep quality is a very

\section{*Corresponding author. Seong Hun Yu (E-mail:} youseonghun@gmail.com)

(C)2015 The Society of Physical Therapy Science. Published by IPEC Inc. This is an open-access article distributed under the terms of the Creative Commons Attribution Non-Commercial No Derivatives (by-ncnd) License $<$ http://creativecommons.org/licenses/by-nc-nd/3.0/> . common problem among aged people, and it is aggravated by aging. The change in the sleeping pattern is a normal phenomenon that takes place with aging, but an altered sleeping pattern sometimes linked to sleep disturbance. As a result, about $14-42 \%$ of elderly people complain of sleep disturbance $^{7)}$.

Sleep disturbance causes physical, social, and psychological weakness among elderly people, affecting their daily life causing decrease in activity, feelings of impotence, a sense of alienation, depression, and feelings of loss. Sleep disturbance can be a risk factor as well as a predisposing factor for elderly people, that triggering fatigue, anxiety, and depression. Sustained sleep disturbance of elderly people sometime actually connected to the development of depression in the elderly ${ }^{8}$.

Regarding the stress that is one of the causes of sleep disturbance, and some empirical studies of elderly people argue that stress of elderly people tends to increase in the elderly as they have relatively less response to interventions, mediating strategies or competency compared to other age groups. It is known that the magnitude of impact from this kind of stress differs according to cognitive function of each individual ${ }^{9}$.

As the most effective means of preventing elderly people's psychological problems caused by aging, such as depression, life stress, and lowered self-esteem, physical 
activity and aerobic exercise of mid-level intensity have received attention ${ }^{10)}$. Regular aerobic exercise has many advantages for the maintenance or reinforcement of physical strength as it provides the body with proper stimulus. Among various aerobic exercises, walking exercise is effective at burning fat and improving cardiopulmonary function ${ }^{11)}$, and it poses little risk of injury because has minor impacts on the joints. Hence, it is often recommended as an effective exercise for the obese. Moreover, walking, which is a rhythmic and dynamic aerobic exercise of sufficient intensity, does not only provides a practical way of promoting health and improving cardiopulmonary function, but also known to be very effective at burning fat. Accordingly, many people practice walking these days. Moreover, diverse types of walking have been recently introduced. Nordic walking is one of them. Nordic walking moves all the muscles in the upper body during walking, including the arms, shoulders, chest, back, etc., in addition to lower body exercise. It also consumes approximately 30-70\% more energy than general walking. Moreover, it puts less pressure on the knee and joints and has a relatively low risk of injury since poles are held by both hands. As it has been reported to be an exercise suitable for the old and infirm, the number of people participating in Nordic walking is increasing ${ }^{12)}$. Moreover, as Nordic walking does not require high technique and it is also inexpensive, making participation easy for everybody. The use of poles activates muscles in the upper body and regular practice increases the walking speed and stride. Nordic walking has been reported to be effective at enhancing the cardiopulmonary functions of healthy individuals ${ }^{13)}$.

A study that compared walking with Nordic walking reported that Nordic walking is more effective at reducing body fat, weight, and body fat percentage, as it increases use of adipose tissues, than to general walking ${ }^{14)}$. Another study reported that for preventing incidents rate following functional deterioration of aged persons, it is necessary to consider the factors such as brisk walking, gender, BMI, depression, and age ${ }^{15)}$.

As such, since Nordic walking consumes more energy efficiently than general walking for the same time spent, should be able to satisfy the diverse needs of busy modern people. Even though there are a number of recent studies that have examined the effects of physical symptoms of Nordic exercise, the studies that have investigated its impact on depression and sleep disturbance are comparatively rare as of now.

Hence, this study analyzed the impacts on Nordic walking and general walking on psychological and physical health aspects through an 8-week training program, to determine whether or not Nordic walking is a healthy exercise that can ameliorate elderly people's depression and sleep disturbance. A secondary objective was to provide ordinary people with walking difficulties with information about enjoying aerobic exercise from the aspect of mental health.

\section{SUBJECTS AND METHODS}

Elderly people who were diagnosed with depression at Center G located in Metropolitan City G in Korea were selected as research subjects. All the subjects provided their informed consent before participating in this study, which was approved by the Dong Shin University Faculty of Health Sciences Human Ethics Committee. Prior to their experiment participation in this study, the subjects listened to an explanation about the purpose, method, and procedure of the experiment, after which they provided their consent to participation. A total of 24 subjects with depression accompanied by sleep disturbance were evenly divided into the Nordic walking group and general walking group by random assignment. The inclusion criteria for participation in this study were: those of depression with Beck depression inventory (BDI) score over 18; those with sleep disturbance with Pittsburgh sleep quality index (PSQI) score over 5; the ability to perform on regular exercise without of the nervous system or the circulatory on system; and sufficient cognitive ability to be able to learn the basic techniques required for walking, to Nordic walking and actually perform them. The subjects had a (mean \pm standard deviation) age of $75.20 \pm 6.25$ year, a height of $161.04 \pm 9.36 \mathrm{~cm}$, and weighed $60.70 \pm 11.09 \mathrm{~kg}$ for weight.

The exercise program for the two groups followed the sequence of cooling, main exercise, and cooling-down. During warming-up, stretching of muscles related to walking exercise was performed for ten times in three sets for each muscle. The cardiovascular endurance of each individual was measured using a wireless heart rate monitor to determine the target heart rate, and the subject followed the exercise program aiming at the target heart rate ${ }^{16)}$. The intervention lasted a total of eight weeks, during which the exercise was performed three times a week at the same time of the day. The 50-minute exercise consisted of 10 minutes of warmingup, 30 minutes of main exercise, and 10 minutes of cooling. The exercise intensity was set at $40 \%$ of what during the early phase of weeks $1-4,60 \%$ during weeks $5-6$, and $75 \%$ during weeks $7-8$. This exercise program was applied to the Nordic walking group and general walking group followed the same exercise program.

The specific exercise rules of Nordic walking were as follows. First, the poles were held up maintained at about $70 \%$ of height (at the height of navel when of standing straight) and stride length was kept at about $100 \mathrm{~cm}$. Second, the subject held the poles lightly as if holding an egg, and subject completely unfolded the palm that held the pole and stretches the heel uprightly when pole passed the hip. Third, the subjects kept the upper body straight without swinging it with the poles' movements ${ }^{12,13)}$.

Depression was evaluated using Beck Depression Inventory (BDI) which can be tested in a short time at relatively low cost. BDI also has the advantage of a simple testing and scoring method, a relatively high reliability, validity, and sensitivity. BDI consists of a total of 21 questions, each of which has four responses indicating intensity of feeling, and that the participants can choose the best one describes themselves. Those with scores between $0-17$ were defined as having no depression, and those with scores over 18 were defined as having depression. The validity and reliability of each item in this study, had Cronbach's $\alpha$ values between $0.81-0.089^{17)}$.

Sleep disturbance was evaluated using the Korean version of the Pittsburgh Sleep Quality Index (PSQI-K) which 
consists of items evaluating subjective sleep quality, sleep latency, sleep duration, habitual sleep efficiency, sleep disturbances, use of sleeping medication, and daytime dysfunction. This is a useful test for assessing sleep quality and each item is assessed using a score from $0-3$, making the total scores of the seven items $0-21$. Those with score equal to or higher than 5 were defined as having sleep disturbance ${ }^{18)}$.

Body, height was measured using an extensimeter, and body composition was measured by a body composition analyzer (Inbody 230, Biospace, Korea) measures bioelectrical impedance after the shoes, socks, and accessories have been removed. The measurements were performed with subjects standing upright with their feet properly covering the electrodes on the measuring stage, with their apart, and their hands holding the handle containing electrodes. Using this measuring device, skeletal muscle mass $(\mathrm{kg})$, total weight without fat $(\mathrm{kg})$, BMI $\left(\mathrm{kg} / \mathrm{m}^{2}\right)$, body fat percentage $(\%)$, and basal metabolic rate (kcal) were measured.

The mean (M) and standard deviation (SD) of the measured data in this study were calculated using SPSS ver 18.0. For Windows. One-way repeated measures ANOVA determine the significance of changes between the groups with the measurement time, and one-way ANOVA was performed to determine within-group time differences. Tukey's test was used as the post-hoc test. The independent t-test was used to test the significance of between-group differences at each measurement time. A significance level of $\alpha=0.05$ was used in all analyses.

\section{RESULTS}

The effects of the exercise program on the elderly with depression are shown in Table 1. Depression showed significant main effects of time in both groups $(p<0.001)$. The Nordic Walking group showed significant differences in depression between before the intervention and after four weeks of the intervention, and between before the intervention and after eight weeks of the intervention $(p<0.001)$. The general walking group also showed significant differences at the same times as the Nordic walking group significant differences $(p<0.001)$ between the two groups were found after four and eight weeks of the intervention $(p<0.05)$.
Sleep quality showed a significant main effect of time $(p<0.001)$ and interaction $(p<0.001)$. The Nordic walking group showed significant differences in sleep quality between before the intervention and after four weeks of the intervention, and between before the intervention and after eight weeks of the intervention $(\mathrm{p}<0.001)$. The general walking group, showed significant differences at the same times as the Nordic walking group $(\mathrm{p}<0.05)$. The two groups showed a significant difference after eight weeks of the intervention $(\mathrm{p}<0.05)$.

The analyses of physical factors are shown in Table 2 . Skeletal muscle showed a significant main effect of time and interaction $(\mathrm{p}<0.001)$, and significant difference between two groups at eight weeks of the intervention. No other significant differences were found.

Body fat percentage, body mass index, and basal metabolism showed significant main effects of time $(p<0.05)$ and interaction $(\mathrm{p}<0.001)$.

\section{DISCUSSION}

Nordic walking has the advantage over general walking of using more muscles, higher calorie consumption, and pole support for the spine, knee, and upper body ${ }^{19}$. Although Nordic walking is not popular in Korea, it has significant potential as an effective physical activity. Moreover, it is

Table 1. Depression and sleep disturbance scores of the Nordic walking and general walking groups

\begin{tabular}{|c|c|c|c|c|}
\hline & & Before & 4 weeks & 8 weeks \\
\hline \multirow[t]{2}{*}{ BDI } & $\mathrm{NWT}^{\mathrm{a}}$ & $24.5 \pm 4.2$ & $14.6 \pm 2.6 \%$ & $11.4 \pm 2.3 * * \div$ \\
\hline & $\mathrm{GWT}^{\mathrm{b}}$ & $24.4 \pm 4.3$ & $17.7 \pm 3.7 \dagger t$ & $13.9 \pm 2.1 * * \dagger+$ \\
\hline \multirow[t]{2}{*}{ PSQI-K } & $\mathrm{NWT}^{\mathrm{c}}$ & $12.6 \pm 3.3$ & $5.4 \pm 1.5 \dagger$ & $4.6 \pm 1.8 * *+$ \\
\hline & GWT & $12.3 \pm 3.0$ & $8.5 \pm 3.0 t$ & $7.6 \pm 3.3^{*+t}$ \\
\hline
\end{tabular}

${ }^{\mathrm{a} N o r d i c}$ walking training group, ${ }^{\mathrm{b}}$ General walking training group, ${ }^{\mathrm{c}}$ Group $\times$ Time interaction

Significant difference among the three times, ${ }^{*} \mathrm{p}<0.05, * * \mathrm{p}$ $<0.001$.

Significant difference between the groups, ${ }^{\dagger} \mathrm{p}<0.05$.

Significant difference from baseline, ${ }^{*} \mathrm{p}<0.001$.

Table 2. Body composition parameters of the Nordic walking and general walking groups

\begin{tabular}{lcccc}
\hline & & Before & 4 weeks & 8 weeks \\
\hline Skeletal muscle & NWT $^{\mathrm{a}}$ & $27.9 \pm 3.7$ & $30.5 \pm 5.8$ & $35.9 \pm 7.5$ \\
mass (kg) & $\mathrm{GWT}^{\mathrm{b}}$ & $28.8 \pm 3.7$ & $28.5 \pm 3.9$ & $28.9 \pm 4.2 \dagger$ \\
Fat free mass & $\mathrm{NWT}^{\mathrm{a}}$ & $50.7 \pm 6.6$ & $52.6 \pm 5.0$ & $54.7 \pm 5.5$ \\
$(\mathrm{~kg})$ & $\mathrm{GWT}^{\mathrm{b}}$ & $51.9 \pm 6.3$ & $51.3 \pm 6.4$ & $51.5 \pm 6.2$ \\
$\mathrm{BMI}$ & $\mathrm{NWT}^{\mathrm{a}}$ & $24.9 \pm 2.7$ & $23.1 \pm 2.6$ & $21.8 \pm 1.9$ \\
$\left(\mathrm{~kg} / \mathrm{m}^{2}\right)$ & $\mathrm{GWT}^{\mathrm{b}}$ & $22.6 \pm 3.3$ & $22.6 \pm 3.6$ & $22.3 \pm 2.7$ \\
Percent body fat & $\mathrm{NWT}^{\mathrm{a}}$ & $25.6 \pm 6.7$ & $22.4 \pm 5.8$ & $20.1 \pm 5.1$ \\
$(\%)$ & $\mathrm{GWT}^{\mathrm{b}}$ & $21.8 \pm 6.4$ & $22.4 \pm 6.6$ & $21.8 \pm 5.7$ \\
Basal metabolic & $\mathrm{NWT}^{\mathrm{a}}$ & $1,434.8 \pm 113.8$ & $1,503.9 \pm 103.1$ & $1,557.4 \pm 104.5$ \\
rate (kcal) & $\mathrm{GWT}^{\mathrm{b}}$ & $1,493.4 \pm 137.9$ & $1,477.5 \pm 137.0$ & $1,532.1 \pm 108.6$ \\
\hline
\end{tabular}

${ }^{\mathrm{a}}$ Nordic walking training group, ${ }^{\mathrm{b}} \mathrm{General}$ walking training group

Significant difference between the groups, ${ }^{\dagger} \mathrm{p}<0.05$. 
reported that participation in Nordic walking group and programs is relatively high, compared to general walking ${ }^{20)}$.

In a previous study that compared treadmill walking between a general walking group and a Nordic walking group that Nordic walking had a positive impact on walking disturbances ${ }^{21)}$. The fundamental and physiological difference between Nordic walking and general walking is that Nordic walking makes use of the upper body muscles and stimulates circulation due to the characteristic of the exercise ${ }^{22)}$.

This study examined how Nordic walking and general walking affect depression, sleep quality, and body composition among elderly people with depression. Depression showed, significant changes with time and a between-group difference was found between Nordic walking and general walking groups. The results regarding the changes in depression are consistent with previous studies that have examined the impact of regular walking exercise on metabolic syndrome, cardiovascular risk, and depression severity among elderly diabetes patients ${ }^{23}$. It is conjectured that elderly people who do not perform regular exercise have a higher likelihood of developing depression than those who regularly exercise. Also, the elderly people who do regular exercise show better depression scores than those who do not regularly exercise. This suggests that regular Nordic walking exercise would be more effective than general walking.

In a previous study of depression, it was argued that cognitive therapy, physical therapy, and functional exercise elicited significant improvements in a rehabilitation program for depression patients with stroke ${ }^{24)}$. It is our belief that Nordic exercise should be included in the rehabilitation program, as a new exercise method for treating the depression of patients with stroke.

Sleep disturbance showed significant changes with time and between-group differences. In the literature on the impact of Nordic walking on sleep disturbance, one study reported that six weeks of aerobic exercise elicited significant improvements in pain, sleep quality and physical function of fibromyalgia syndrome patients with sleep disturbance ${ }^{25)}$, consistent with the present study's results.

This implies that regular exercise can ameliorate depression and sleep disturbance that accompanying chronic diseases such as diabetes and nephritis. There are a variety of biological and psychological hypotheses regarding the positive effects of participation in regular exercise on psychological conditions, for example: the thermogenic hypothesis, endorphin hypothesis, distraction hypothesis, and mastery hypothesis. However, these hypotheses do not consistently predict the psychological effects of exercise, and there is currently no fully verified hypothesis regarding this point. Hence, continuous verification efforts will be necessary ${ }^{26)}$. Nevertheless, as was shown by this study, an exercise program of 8 weeks of Nordic walking can provide psychological stability indicating the possibility of Nordic walking as an alternative exercise in the treatment of elderly people's depression and sleep disturbance.

Skeletal muscle mass showed a main effect of time and interaction between the Nordic walking group and general walking group. We attribute this to the pole use in Nordic walking, in which the upper body moves freely, and moving the poles has a positive effect on skeletal muscle mass, suggesting of Nordic walking is more effective than other physical activities.

In this study, variables of total weight without fat, BMI, body fat percentage, and basal metabolic rate showed main effects of time and group interaction. Fat-free mass showed numerical changes due to Nordic walking, but the differences were not significant. It is our opinion that a significant effect would be obtained if the exercise were prolonged period. Similarly, the absence of significant differences in BMI, body fat percentage, and basal metabolic rate in the Nordic walking group can be attributed to the relatively short exercise period and an uncontrolled diet. Amount of exercise, qualitative factors, and the absence of a control group are additional reasons for the lack of significant results. In order to address the issue of the absence of a control group, the short exercise period, and relatively weak exercise intensity, future studies should include a control group to thoroughly conduct comparative analyses on diverse quantitative and qualitative factors. Moreover, there were individual variations in pole lengths and weights. Considering that this could be an important factor in determining the effects of the exercise, future studies should also address this issue. In summary, Nordic walking for eight weeks ameliorated depression and sleep disturbance of the elderly subjects with depression, indicating that Nordic walking is an exercise that is effective for treating sleep disturbance.

\section{REFERENCES}

1) Baltes P, Mayer K: The Berlin Aging Study. Cambridge University Press, 2001.

2) Schulz R, Drayer RA, Rollman BL: Depression as a risk factor for non-suicide mortality in the elderly. Biol Psychiatry, 2002, 52: 205-225. [Medline]

3) Waern M, Rubenowitz E, Wilhelmson K: Predictors of suicide in the old elderly. Gerontology, 2003, 49: 328-334. [Medline] [CrossRef]

4) Bolger N, DeLongis A, Kessler RC, et al.: Effects of daily stress on negative mood. J Pers Soc Psychol, 1989, 57: 808-818. [Medline]

5) Wu B, Chi I, Plassman BL, et al.: Depressive symptoms and health problems among Chinese immigrant elders in the US and Chinese elders in China. Aging Ment Health, 2010, 14: 695-704. [Medline] [CrossRef]

6) Eser I, Khorshid L, Cinar S: Sleep quality of older adults in nursing homes in Turkey: enhancing the quality of sleep improves quality of life. J Gerontol Nurs, 2007, 33: 42-49. [Medline]

7) Elwood P, Hack M, Pickering J, et al.: Sleep disturbance, stroke, and heart disease events: evidence from the Caerphilly cohort. J Epidemiol Community Health, 2006, 60: 69-73. [Medline]

8) Livingston G, Blizard B, Mann A: Does sleep disturbance predict depression in elderly people? A study in inner London. Br J Gen Pract, 1993, 43: 445-448. [Medline]

9) Jeste DV, Depp CA, Vahia IV: Successful cognitive and emotional aging. World Psychiatry, 2010, 9: 78-84. [Medline]

10) Windle G, Hughes $D$, Linck $P$, et al.: Is exercise effective in promoting mental well-being in older age? A systematic review. Aging Ment Health, 2010, 14: 652-669. [Medline] [CrossRef]

11) Ahn JM, Kim SW, Kang JH, et al.: Depression and weight loss in low calorie diet and low calorie diet with exercise on obese women, J korean Acad. Fam Med, 1999, 20: 1239-1246.

12) Schiffer T, Knicker A, Hoffman U, et al.: Physiological responses to nordic walking, walking and jogging. Eur J Appl Physiol, 2006, 98: 56-61. [Medline] [CrossRef]

13) Morsø L, Hartvigsen J, Puggaard L, et al.: Nordic walking and chronic low back pain: design of a randomized clinical trial. BMC Musculoskelet Disord, 2006, 7: 77. [Medline] [CrossRef]

14) Horowitz JF: Fatty acid mobilization from adipose tissue during exercise. Trends Endocrinol Metab, 2003, 14: 386-392. [Medline] [CrossRef]

15) Chalé-Rush A, Guralnik JM, Walkup MP, et al.: Relationship between physical functioning and physical activity in the lifestyle interventions and independence for elders pilot. J Am Geriatr Soc, 2010, 58: 1918-1924. 
[Medline] [CrossRef]

16) Kim SH: Effects of nordic walking exercise on bone density and bone relation factors in elderly women. Korea J Phys Education, 2013, 52: 571-584

17) Beck AT, Ward CH, Mendelson M, et al.: An inventory for measuring depression. Arch Gen Psychiatry, 1961, 4: 561-571. [Medline]

18) Sohn SI, Kim H, Lee MY, et al.: The reliability and validity of the Korean version of the Pittsburgh Sleep Quality Index. Sleep Breath, 2012, 16 803-812. [Medline] [CrossRef]

19) Shove E, Pantzar M: Consumers, producers and practices: understanding the invention and reinvention of Nordic Walking. J Consum Cult, 2005, 5: $43-64$.

20) Figard-Fabre H, Fabre N, Leonardi A, et al.: Efficacy of Nordic walking in obesity management. Int J Sports Med, 2011, 32: 407-414. [Medline]

21) Shim JM: Comparison of gait and feet during Nordic pole walking and unassisted walking on a treadmill. J Phys Ther Sci, 2012, 24: 1225-1228.

22) Figard-Fabre $\mathrm{H}$, Fabre N, Leonardi A, et al.: Physiological and perceptual responses to Nordic walking in obese middle-aged women in comparison with the normal walk. Eur J Appl Physiol, 2010, 108: 1141-1151. [Medline] [CrossRef]

23) Sung KW, Lee JH: The effects of regular walking exercise on metabolic sundrome, cardiovascular risk factor, and depressive symptoms in the elderly with diabetic mellitus. J Korean Acad Community Health Nurs, 2010, 21: 409-418. [CrossRef]

24) Jeong YJ, Kim WC, Kim YS, et al.: The relationship between rehabilitation and changes in depression in stroke patients. J Phys Ther Sci, 2014, 26: 1263-1266. [Medline] [CrossRef]

25) Vural M, Berkol TD, Erdogdu Z, et al.: Evaluation of the effectiveness of an aerobic exercise program and the personality characteristics of patients with fibromyalgia syndrome: a pilot study. J Phys Ther Sci, 2014, 26 : 1561-1565. [Medline]

26) Salmon P: Effects of physical exercise on anxiety, depression, and sensitivity to stress: a unifying theory. Clin Psychol Rev, 2001, 21: 33-61. [Medline] [CrossRef] 